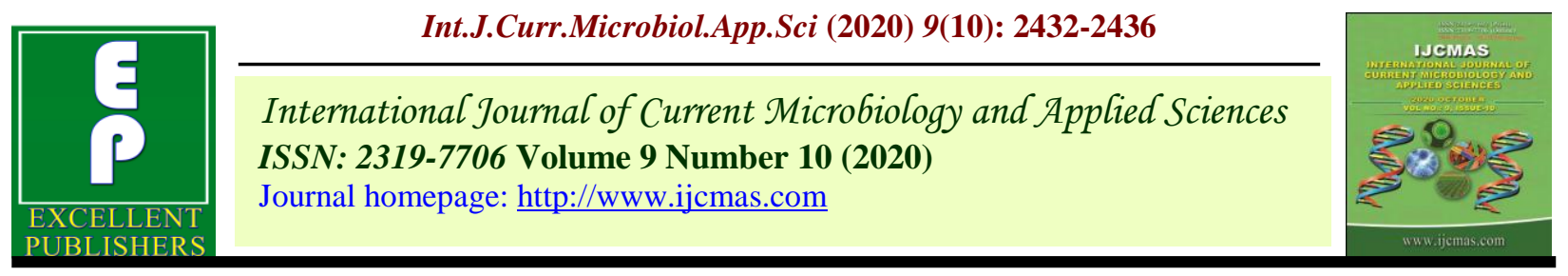

Original Research Article

https://doi.org/10.20546/ijcmas.2020.910.290

\title{
Effect of Soil and Foliar Application of Nutrients on Pre-harvest Studies of Cluster Bean
}

\author{
Kampoter*, Raj Pal Singh Tomar, Chandrabhan Singh Jatav and Ramkesh Patel \\ Department of Agronomy, RVKVV, Gwalior, India \\ *Corresponding author
}

\section{A B S T R A C T}

\begin{tabular}{l} 
Ke y w or d s \\
$\begin{array}{l}\text { Soil, Foliar, } \\
\text { Nutrient and cluster } \\
\text { bean }\end{array}$ \\
Article Info \\
$\begin{array}{l}\text { Accepted: } \\
\text { 17 September } 2020 \\
\text { Available Online: } \\
10 \text { October } 2020\end{array}$ \\
\hline
\end{tabular}

\section{Introduction}

Cluster bean (Cyamopsis tetragonoloba L. Taub) commonly known as Guar, is a drought and high temperature tolerant, deep rooted Kharif annual legume of high social and economic significance. It is highly adaptable towards erratic rainfall have multiple industrial uses and it is one of the most significant crop in cropping system for farmers of arid areas. Guar is also grown in other parts of the world, like, Pakistan, USA, Australia, Brazil and South Africa. In India the productivity of cluster bean is very low. So, there is need to take proper agronomic practices to enhance the productivity of cluster bean and foremost important among them is soil and foliar application of organic and inorganic sources of nutrients exploiting genetic potential of crop. This is considered to be an efficient and economic method of supplementing part of nutrient requirement at critical growth stages of the crop. Foliar application is credited with the advantage of quick and efficient utilization of nutrients, elimination of losses through leaching, fixation and regulating uptake of nutrients by the plant. Since foliar nutrients usually penetrate the leaf cuticle or stomata and enters the cell facilitating easy and rapid utilization of nutrients. So, soil and foliar nutrition on cluster bean helps in achieving the optimum grain yield of cluster bean. 


\section{Materials and Methods}

The experiment was conducted on the Research Farm, College of Agriculture, Gwalior (M.P.). The topography of the field was uniform with proper drainage. The experiment was laid out in the randomized block design with 10 treatments and each treatment was replicated three times. The following treatment combinations involving as a soil application of PSB and KMB while foliar spray of NAA, MOP, DAP, NPK (18:18:18), zinc sulphate, thiourea, neem coated urea and salicylic acid were applied.

\section{Results and Discussion}

Soil and foliar application of nutrients caused a marked variation in growth parameters of cluster bean at all of the crop growth stages. The growth parameters viz., plant height, number of branches per plant, number of leaves per plant and dry weight per plant had a direct relationship with soil and foliar application of nutrients. All soil and foliar nutrient treatments significantly increased all growth parameters over control treatment $\left(\mathrm{T}_{1}\right)$.

The maximum values of all these growth parameters were recorded with the NPK (18:18:18) $1 \%$ spray at flower initiation and 10 days after $1^{\text {st }}$ spray $\left(\mathrm{T}_{5}\right)$ followed by $\mathrm{T}_{10}$ (Neem coated urea $1 \%$ + salicylic acid 75 ppm spray at flower initiation and 10 days after $1^{\text {st }}$ spray), $\mathrm{T}_{9}(\mathrm{PSB}+\mathrm{KMB}$ soil applied + NAA $20 \mathrm{ppm}$ spray at flower initiation stage) and $\mathrm{T}_{2}$ (PSB soil applied + NAA $20 \mathrm{ppm}$ spray at flower initiation stage) and these treatments were found significantly superior over other treatments.

The order of significance were followed by application of KMB soil applied + NAA 20 ppm spray at flower initiation stage $\left(\mathrm{T}_{7}\right)$, DAP $0.5 \%$ spray at flower initiation and 10 days after $1^{\text {st }}$ spray $\left(\mathrm{T}_{3}\right)$ Thiourea @ 500 ppm spray at vegetative and flowering stage $\left(\mathrm{T}_{8}\right)$ Zinc sulphate $0.25 \%$ spray at flower initiation and 10 days after $1^{\text {st }}$ spray $\left(\mathrm{T}_{6}\right)$ and MOP $0.5 \%$ spray at flower initiation and 10 days after $1^{\text {st }}$ spray $\left(\mathrm{T}_{4}\right)$.

Similar results had also been reported by Sujatha (2001) reported that foliar application of salicylic acid (100 ppm) on green gram at 75 DAS increased plant height $(50.4 \mathrm{~cm})$, root length $(16.9 \mathrm{~cm})$, number of leaves (18.4) and Leaf area index (LAI) (1.30).

Mona and Azab (2016) reported that the effects of foliar application of NPK compound with $\mathrm{Fe}, \mathrm{Zn}$ and $\mathrm{Mn}$ at different doses on cowpea plants. In addition, soluble fertilizers NPK (19:19:19) and $500 \mathrm{ppm} \mathrm{Fe}$, $300 \mathrm{ppm} \mathrm{Zn}$ and $300 \mathrm{ppm} \mathrm{Mn}$ were applied. Four treatments of fertilization were tested: control (no fertilization), (50\%), (100\%), and $(125 \%)$.

The NPK fertilizers were sprayed every 15 days. The results are as follows: Foliar fertilization NPK with $\mathrm{Fe}, \mathrm{Zn}$ and Mn reflect increasement in vegetative growth, yield and its components and nutrient concentration of cowpea plant compared with control.

Prajapati and Modi (2016) conducted an experiment under hydroponics condition using micronutrient containing nutrient solution to evaluate the effect of potassium solubilizing bacteria KSB-8 (Enterobactor hormaechei), the results indicated that a remarkable increase in root length, flowering, fruit setting, fruit maturing, $\mathrm{K}$ content and chlorophyll content. Thus, it might be concluded that KSB-8 (Enterobactor hormaechei) could be used as crop-enhancer and bio-fertilizer for cucumber (Cucumis sativas) and other $\mathrm{K}$ rich crops under hydroponic condition (Table 1 and 2). 
Table.1

\begin{tabular}{|c|c|c|c|c|c|c|}
\hline \multirow[t]{2}{*}{ Treatments } & \multicolumn{2}{|c|}{$\begin{array}{l}\text { Plant population/m row } \\
\text { length }\end{array}$} & \multicolumn{4}{|c|}{ Plant height (cm) } \\
\hline & Initial & Final & 30 DAS & 60 DAS & 90 DAS & Harvest \\
\hline $\mathbf{T}_{1:}$ Control (water spray). & 10.43 & 9.86 & 20.13 & 76.18 & 85.70 & 86.07 \\
\hline $\begin{array}{l}\mathrm{T}_{2} \text { : PSB soil applied + NAA } 20 \text { ppm spray at flower } \\
\text { initiation stage. }\end{array}$ & 10.53 & 10.23 & 22.37 & 86.40 & 98.50 & 101.27 \\
\hline $\begin{array}{l}T_{3}: \text { DAP } 0.5 \% \text { spray at flower initiation and } 10 \\
\text { days after } 1^{\text {st }} \text { spray. }\end{array}$ & 10.64 & 9.66 & 20.00 & 80.47 & 91.50 & 93.16 \\
\hline $\begin{array}{l}\mathrm{T}_{4}: \text { MOP } 0.5 \% \text { spray at flower initiation and } 10 \\
\text { days after } 1^{\text {st }} \text { spray. }\end{array}$ & 10.21 & 9.32 & 19.07 & 78.03 & 88.13 & 89.23 \\
\hline $\begin{array}{l}T_{5}: \text { NPK }(18: 18: 18) 1 \% \text { spray at flower initiation and } \\
10 \text { days after } 1^{\text {st }} \text { spray. }\end{array}$ & 10.43 & 9.69 & 20.17 & 94.13 & 106.39 & 108.00 \\
\hline $\begin{array}{l}T_{6}: \text { Zinc sulphate } 0.25 \% \text { spray at flower initiation } \\
\text { and } 10 \text { days after } 1^{\text {st }} \text { spray. }\end{array}$ & 10.40 & 10.10 & 19.53 & 78.07 & 88.73 & 90.30 \\
\hline $\begin{array}{l}T_{7}: \text { KMB soil applied + NAA } 20 \text { ppm spray at } \\
\text { flower initiation stage. }\end{array}$ & 10.24 & 9.67 & 21.17 & 84.80 & 95.53 & 98.47 \\
\hline $\begin{array}{l}T_{8} \text { : Thiourea @ } 500 \text { ppm spray at vegetative and } \\
\text { flowering stage. }\end{array}$ & 10.23 & 9.99 & 21.03 & 79.73 & 91.03 & 92.97 \\
\hline $\begin{array}{l}\text { T9: PSB + KMB soil applied + NAA } 20 \text { ppm spray at } \\
\text { flower initiation stage. }\end{array}$ & 10.63 & 10.26 & 23.47 & 86.97 & 99.00 & 102.27 \\
\hline $\begin{array}{l}T_{10}: \text { Neem coated urea } 1 \%+\text { salicylic acid } 75 p p m \\
\text { spray at flower initiation and } 10 \text { days after } 1^{\text {st }} \text { spray. }\end{array}$ & 10.35 & 10.03 & 20.37 & 91.33 & 103.32 & 106.35 \\
\hline S.E.(m) \pm & 0.202 & 0.358 & 0.757 & 2.751 & 3.367 & 2.399 \\
\hline C.D.(at 5\%) & NS & NS & 2.250 & 8.174 & 10.004 & 7.128 \\
\hline
\end{tabular}


Table.2

\begin{tabular}{|c|c|c|c|c|c|c|c|c|c|c|c|c|}
\hline \multirow[b]{2}{*}{ Treatments } & \multicolumn{4}{|c|}{ Number of branches/plant } & \multicolumn{4}{|c|}{ Number of leaves/plant } & \multicolumn{4}{|c|}{ Plant dry weight (g) } \\
\hline & $\begin{array}{c}30 \\
\text { DAS }\end{array}$ & $\begin{array}{c}\text { 60 } \\
\text { DAS }\end{array}$ & $\begin{array}{c}90 \\
\text { DAS }\end{array}$ & $\begin{array}{c}\text { Harv } \\
\text { est }\end{array}$ & $\begin{array}{c}\text { 30 } \\
\text { DAS }\end{array}$ & $\begin{array}{c}\text { 60 } \\
\text { DAS }\end{array}$ & $\begin{array}{c}90 \\
\text { DAS }\end{array}$ & $\begin{array}{c}\text { Harv } \\
\text { est }\end{array}$ & $\begin{array}{c}\text { 30 } \\
\text { DAS }\end{array}$ & $\begin{array}{c}\text { 60 } \\
\text { DAS }\end{array}$ & $\begin{array}{c}90 \\
\text { DAS }\end{array}$ & $\begin{array}{c}\text { Harv } \\
\text { est }\end{array}$ \\
\hline $\mathbf{T}_{1:}$ Control (water spray). & 4.13 & 5.33 & 6.60 & 6.60 & 10.03 & 24.57 & 26.37 & 23.83 & 2.00 & 9.03 & 26.17 & 26.83 \\
\hline $\begin{array}{l}\mathrm{T}_{2} \text { : PSB soil applied + NAA } 20 \mathrm{ppm} \\
\text { spray at flower initiation stage. }\end{array}$ & 4.90 & 7.17 & 8.30 & 8.30 & 11.67 & 32.60 & 38.49 & 34.33 & 2.93 & 12.57 & 35.77 & 38.67 \\
\hline $\begin{array}{l}T_{3} \text { : DAP } 0.5 \% \text { spray at flower } \\
\text { initiation and } 10 \text { days after } 1^{\text {st }} \text { spray. }\end{array}$ & 3.87 & 6.33 & 7.67 & 7.67 & 10.60 & 29.93 & 34.67 & 30.73 & 2.37 & 10.77 & 31.03 & 33.30 \\
\hline $\begin{array}{l}\text { T}_{4} \text { : MOP } 0.5 \% \text { spray at flower } \\
\text { initiation and } 10 \text { days after } 1^{\text {st }} \text { spray. }\end{array}$ & 4.10 & 5.63 & 6.73 & 6.73 & 10.26 & 25.53 & 31.30 & 27.27 & 2.23 & 9.83 & 27.33 & 28.03 \\
\hline $\begin{array}{l}T_{5}: \text { NPK (18:18:18) } 1 \% \text { spray at } \\
\text { flower initiation and } 10 \text { days after } 1^{\text {st }} \\
\text { spray. }\end{array}$ & 4.20 & 7.60 & 8.87 & 8.87 & 10.67 & 35.69 & 40.37 & 36.31 & 2.17 & 14.83 & 38.87 & 41.57 \\
\hline $\begin{array}{l}T_{6}: \text { Zinc sulphate } 0.25 \% \text { spray at } \\
\text { flower initiation and } 10 \text { days after } 1^{\text {st }} \\
\text { spray. }\end{array}$ & 3.97 & 6.13 & 7.27 & 7.27 & 9.93 & 26.43 & 31.43 & 28.93 & 1.93 & 10.07 & 28.27 & 30.17 \\
\hline $\begin{array}{l}\mathrm{T}_{7}: \mathrm{KMB} \text { soil applied + NAA } 20 \text { ppm } \\
\text { spray at flower initiation stage. }\end{array}$ & 4.80 & 6.50 & 7.73 & 7.73 & 11.33 & 31.00 & 36.93 & 33.47 & 2.67 & 12.43 & 33.50 & 35.07 \\
\hline $\begin{array}{l}T_{8}: \text { Thiourea @ } 500 \text { ppm spray at } \\
\text { vegetative and flowering stage. }\end{array}$ & 4.73 & 6.27 & 7.30 & 7.30 & 11.17 & 28.63 & 33.57 & 30.30 & 2.60 & 10.47 & 29.03 & 31.10 \\
\hline $\begin{array}{l}\text { T9: PSB + KMB soil applied + NAA } 20 \\
\text { ppm spray at flower initiation stage. }\end{array}$ & 5.37 & 7.30 & 8.37 & 8.37 & 12.13 & 33.19 & 39.41 & 35.32 & 3.17 & 12.80 & 35.93 & 38.93 \\
\hline $\begin{array}{l}T_{10}: \text { Neem coated urea } 1 \%+\text { salicylic } \\
\text { acid } 75 \mathrm{ppm} \text { spray at flower initiation } \\
\text { and } 10 \text { days after } 1^{\text {st }} \text { spray. }\end{array}$ & 4.53 & 7.50 & 8.40 & 8.40 & 10.80 & 34.81 & 39.79 & 36.26 & 2.53 & 13.81 & 36.57 & 39.53 \\
\hline S.E.(m) \pm & 0.158 & 0.322 & 0.365 & 0.365 & 0.232 & 0.943 & 1.012 & 0.885 & 0.157 & 0.787 & 1.225 & 1.082 \\
\hline C.D.(at 5\%) & 0.471 & 0.956 & 1.085 & 1.085 & 0.689 & 2.803 & 3.006 & 2.629 & 0.466 & 2.340 & 3.639 & 3.215 \\
\hline
\end{tabular}


Dey et al. (2017) conducted a field experiment to find out the influence of Urea, $\mathrm{KCl}, \mathrm{Zn}$ placement and spray on growth of Cowpea crop. Application of various levels of Urea, $\mathrm{KCl}$ and Zinc which significantly increased dry matter production/plant, plant height, number of branches/plant, number of trifoliate/plant, total nodule/plant. Foliar nutrient sprays viz., $2 \%$ urea, $2 \% \mathrm{KCL}, 1.5 \%$ $\mathrm{ZnSO} 4$. Foliar spray treatment with the aqueous solution of nutrients was done to the 15 and 30 DAS of cowpea crop. Significant increase was recorded in plant height, dry matter production, Maximum growth was recorded when spread with $2 \%$ urea spray followed by $2 \% \mathrm{KCl}$ at flowering and 15 days later is the viable nutrient management package to the Cowpea for getting higher income through higher productivity.

The results obtained are also in close conformity with the findings of $\mathrm{Haq}$ and Mallarino (2000), Reddy et al., (2005), Deshmukh et al. (2008) and Afshari et al., (2013).

\section{References}

Afshari, M., Shekari, F., Azimkhani, R., Habibi, H. and Fotokin, M.H. (2013). Effect of foliar application of salicylic acid on growth and physiological attributes of cowpea under water stress conditions. Iran Agricultural Research, Vol. 32, No.1.

Deshmukh, S. G., Kale, H. B. and Solunke, P. S. (2008). Influence of graded fertility levels and urea spray on growth, yield and economics of rajmah. Annals. of Plant Physiol., 22 (2): 189-191.

Dey, Sonu., Prasad, Sant., Tiwari, Prabhat., and Sharma, Prashant. (2017). Effect of urea, $\mathrm{KCl}$, Zinc placement \& spray on growth of cowpea. Journal of Pharmacognosy and Phytochemistry; SP 1: 971-973.

Haq, M.U. and Mallarino, A.P. (2000). Soybean yield and nutrient composition as affected by early season foliar fertilization. Agron J. 92 (1): 16-24.

Mona, E., Azab, E.,1. (2016). Effects of Foliar NPK Spraying with Micronutrients on Yield and Quality of Cowpea Plants. Asian Journal of Applied Sciences, ISS $N$ : 2321-089.

Prajapati, K. and Modi, H. A. (2016). Growth promoting effect of potassium solubilizing Enterobactor Hormaechei (KSB-8) on Cucumber (Cucumis sativas) under hydroponic condition. Int. J. Adv. Res. Biol. Sci., 3 (5): 168173.

Reddy, M., Padamaja, B., Rao, L.J. and Radhakrishana, K.V. (2005). Effect of foliar spray of urea on nitrogen uptake and yield of urd bean under rainfed condition. Indian J. Dryland Agric. Res. Develop., 20 (2): 151-154.

Sujatha, K.B., (2001). Effect of foliar spray of chemicals and bioregulators on growth and yield of greengram (Vigna radiata L.). M.Sc. Thesis, Tamil Nadu Agric. Univ., Coimbatore.

\section{How to cite this article:}

Kampoter, Raj Pal Singh Tomar, Chandrabhan Singh Jatav and Ramkesh Patel. 2020. Effect of Soil and Foliar Application of Nutrients on Pre-harvest Studies of Cluster Bean. Int.J.Curr.Microbiol.App.Sci. 9(10): 2432-2436. doi: https://doi.org/10.20546/ijcmas.2020.910.290 